\title{
Stefan Berking • Klaus Herrmann \\ Formation and discharge of nematocysts is controlled by a proton gradient across the cyst membrane
}

Received: 4 March 2005/ Revised: 24 October 2005Accepted: 24 October 2005 / Published online: 26 November 2005

(C) Springer-Verlag and AWI 2005

\begin{abstract}
Cnidaria catch and kill their prey by means of nematocysts. A nematocyst consists of a capsule containing a coiled tubule. On triggering, the cyst extrudes this tubule in an extremely rapid manner. The mechanisms and driving forces of discharge are still unknown. We found nematocysts of various cnidarians to be acidic inside and propose that the $\mathrm{pH}$ difference between cyst matrix and cytoplasm drives the discharge of cysts. For large cysts of Aiptasia we calculated that the internal concentration of protons and protonated carboxyl groups is about $5 \mathrm{M}$. Cysts contain polyacids in a high concentration. At a low $\mathrm{pH}$ several of the carboxyl groups of the polymer are uncharged. The carboxyl groups dissociate when, on triggering, the proton concentration becomes balanced across the cyst membrane. The speed of protons in water is extremely rapid. Thus, the equilibration of the proton concentration initially results in a negative net charge within the cyst and therefore in a sudden electrostatic repulsion between the dissociated carboxyl groups of the polymer. This causes an increase in the pressure of the matrix against the cyst wall. We suggest that this nonosmotic pressure increase causes the first and extremely rapid step of discharge. We propose that in a second step cations and water are taken up, generating an increase in osmotic pressure. A change in the $\mathrm{pH}$ value may also facilitate the invagination and evagination, respectively, of the tubule.
\end{abstract}

Keywords Cnidaria $\cdot$ Nematocyte $\cdot$ Polyglutamic acid $\cdot \mathrm{pH}$ gradient $\cdot$ Lysosome

Communicated by H.-D. Franke

S. Berking $(\varangle) \cdot K$. Herrmann

Zoological Institute, University of Cologne,

Weyertal 119, 50923 Köln, Germany

E-mail: s.berking@uni-koeln.de

Tel.: + 49-221-4702248

Fax: + 49-221-4705171

\section{Introduction}

Cnidaria produce stinging cells, termed nematocytes, which are said to house the most complex organelles of animal cells (Fig. 1). These organelles, termed nematocysts, consist of a capsule and a tubule each, both derived from the Golgi apparatus. The tubule grows as an appendix of the capsule by repeated fusion with vesicles at its tip, and is finally taken up by invagination (Schneider 1900; Ewald 1915; both after Kühn 1916). The fully developed cyst is located close to the cell membrane so that a potential prey can trigger its discharge. On discharge the tubules of certain cysts intrude the prey and release toxins that can kill it. Other types of cysts are used for defense or locomotion.

Stenoteles of Hydra are the best-studied nematocysts (for review, see Kass-Simon and Scappaticci 2002, Thurm et al. 2004). The whole process of tubule extrusion is completed within $3 \mathrm{~ms}$. After an electric triggering the volume of the capsule instantaneously increases by more than $10 \%$. Then a preformed opening, the operculum, opens and the stylet-bearing portion of the tubule is ejected. This phase is extremely short, taking less than $10 \mu \mathrm{s}$. Subsequently, after a short resting phase of about $150 \mathrm{~ms}$, the tubule is completely extruded (Tardent and Holstein 1982; Holstein and Tardent 1984).

The mechanisms and driving forces of discharge have been studied by a large number of authors for more than 100 years (for review see Kühn 1916; Bouillon 1993; Kass-Simon and Scappaticci 2002). In general, a high internal osmotic pressure and mechanical energy stored in the walls of cysts and/or tubules have been suggested as causing the discharge when an external cue reaches the cnidocil. The cnidocil is a mechanoreceptor on the cell surface. The observation of Holstein and Tardent (1984) that a stenotele cyst swells immediately before discharge by about $10 \%$ is a particular challenge. The authors proposed that the initial volume increase is produced osmotically, whereas the subsequent discharge 
Fig. 1 Nematocysts of Aiptasia. a The large discharged and undischarged cysts are microbasic mastigophores isolated from (b) acontia of Aiptasia sp. (Anthozoa). Bar $10 \mu \mathrm{m}(\mathbf{a}), 100 \mu \mathrm{m}$ (b)
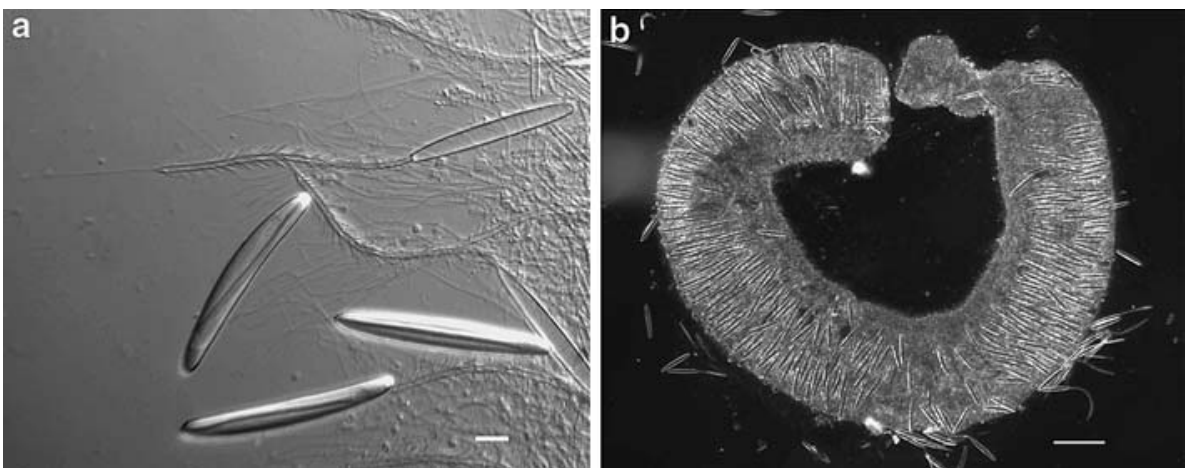

additionally involves the release of mechanical energy stored in the capsular wall. Weber (1989) argued that stenoteles in Hydra are under a constantly high internal osmotic pressure (15.3 $\mathrm{MPa})$. The pressure is dominated by Donnan potential; all small, inorganic ions but not certain large polyanions (polyglutamic acid) are assumed to be able to move unhindered into and out of the capsule. The monomer concentration of the polyglutamic acid was calculated to reach $2 \mathrm{M}$. The $\mathrm{pH}$ was assumed to be almost identical with that of the surrounding cytoplasm (Weber 1989). With respect to various mastigophores of Anemonia viridis, Thomason (1991) proposed the discharge results from an increase of the intracapsular pressure ranging from 0.97 to $1.9 \mathrm{MPa}$

However, most authors claim there is no convincing explanation of how the osmotic pressure can suddenly increase. Indeed, it is difficult to understand how a cyst that is in equilibrium with its surroundings can swell on triggering. Exocytosis (e.g., see Holstein and Tardent 1984; Golz 1994; Gitter and Thurm 1996), that is, the fusion of the cyst membrane with the cell membrane as the initial step of discharge, results in a net loss of the highly concentrated compounds. One would expect the osmotic pressure to decrease, rather than to increase initially. Furthermore, the idea that the discharge is driven by stored mechanical energy fits the volume decrease observed as a second step of the discharge of stenoteles (Tardent and Holstein 1982) but does not fit the initial volume increase. On discharge the volume of several types of cysts decreases only slightly, whereas in some cases it even increases (Lipin 1910, after Kühn 1916). However, if the energy is stored in the coiled tubule (Kass-Simon and Scappaticci 2002), one confronts the problem of explaining how such a tubule is taken up by the capsule and how a volume decrease of certain cysts (Kühn 1916) is obtained when such a cyst, including its invaginated tubule, takes its final step of development while already positioned in a tentacle. These obvious problems led Ewald (1915, after Kühn 1916) to propose that on triggering, compounds of the matrix change their state in such a way that they need a higher volume: "eine Substanz von labiler $\mathrm{Zu}$ sammensetzung," [die] "ihre Konstitution verändert und dadurch einen größeren Raum einnimmt" (p. 474). This change of state would result in a pressure against the cyst wall and could initiate the discharge. For a complete discharge, Ewald proposed, water is taken up, causing further swelling of the matrix. We suggest that precisely this takes place.

We suggest that the energy for the initial swelling and subsequent discharge is stored in a proton gradient across the cyst wall. Triggering may cause the release of protons, which results in an immediate dissociation of carboxyl groups of the stored polyacids in the cyst matrix. The resultant negative charge would cause an electrostatic repulsion among the carboxyl groups, followed by an increase in volume and the first step of discharge. Subsequently, water and cations may be taken up by the cyst, causing an increase of the osmotic pressure. The altered $\mathrm{pH}$ may affect components of the tubule wall in such a way that the evagination of the tubule is favored.

\section{Materials and methods}

\section{Animals}

Polyps of Aiptasia sp. (Anthozoa), Aurelia aurita (Scyphozoa), Clytia johnstoni (Hydrozoa), Thecocodium quadratum (Hydrozoa), Cladonema sp. (Hydrozoa), and Tripedalia cystophora (Cubozoa) were cultured at $20^{\circ} \mathrm{C}$ in artificial seawater of 1050 mosmol (Tagis Tropic Marine, Dreieich, Germany). In experiments with medusae, only freshly released specimens were used. Hydra vulgaris (strain Zürich) was cultured in buffered artificial fresh water at $20^{\circ} \mathrm{C}$ (Zeretzke et al. 2002).

\section{$\mathrm{pH}$ measurements}

Acontia of Aiptasia sp. were isolated mechanically and placed on the top of a FET pH-meter (Shindengen, Electric MFG Co, Tokyo, Japan). Then seawater was removed down to a volume of about $15 \mu \mathrm{l}$. Finally, $40 \mu \mathrm{l}$ of distilled water was added. This treatment resulted in a discharge of cysts. Polyps of A. aurita were induced to produce medusae (strobilation), according to Kroiher et al. (2000). Only medusae (ephyrae) that dropped off 
were put on the FET, the amount of seawater was reduced down to a volume of about $15 \mu \mathrm{l}$, and distilled water was added. Spontaneously produced medusae of $C$. johnstoni, Cladonema sp., and $T$. quadratum were treated in the same way. Polyps of $H$. vulgaris (strain Zürich) were taken from a mass culture and placed on the FET. After removing culture medium to about $15 \mu \mathrm{l}$, saturated $\mathrm{NaCl}$ (in culture medium, 10-20 $\mu$ l) was added to cause nematocyst discharge (Will 1914, after Kühn 1916).

\section{Staining}

Fixed and also living animals were stained with Acridin orange and DAPI (4',6-diamidino-2-phenylindole), respectively, according to Szczepanek et al. (2002). Animals were fixed in 4\% formaldehyde. Further, living animals were treated with LysoSensor (TM) Blue DND167 (Molecular Probes) dissolved in dimethylsulfoxide (DMSO) as described in the distributor's instructions. Poly-DL-aspartic acid, sodium salt $40 \mathrm{wt} \%$ solution in water (Sigma), was mixed 1:1 with concentrated $\mathrm{HCl}$. The precipitate was washed two times with concentrated $\mathrm{HCl}$ and then transferred to diluted $\mathrm{HCl}$. CM-Sephadex C-25 (Pharmacia) was equilibrated with $0.5 \mathrm{M}$ sodium phosphate buffer at $\mathrm{pH} 3.0$ and $\mathrm{pH} 4.0$, respectively. Microscopic observations and photographs were made using an Axiocam camera and an Axioskop fluorescence microscope (Zeiss) equipped with a UV-filterset (Ex $365 \mathrm{~nm}, \mathrm{Em}$ 445/50 nm) and an FITC/GFP filterset (Ex $470 / 40 \mathrm{~nm}$, Em 525/50 nm).

\section{Results}

The marine Anthozoon Aiptasia sp. contains extremely large nematocysts (up to $60 \mu \mathrm{m}$ in length) within special organs (Fig. 1). These organs, termed acontia, are thin filaments of the gastric epithelium. On mechanical contact they are expelled through the mouth and through openings in the gastric wall. Such acontia were removed with the help of scissors, cut into pieces of about $3 \mathrm{~mm}$ length, and placed on top of a FET $\mathrm{pH}$-meter in a volume of about $15 \mu \mathrm{l}$ seawater. When distilled water was added $(40 \mu \mathrm{l})$ several cysts discharged, and at the same time the $\mathrm{pH}$ decreased from 7.8 to minimally 6.4
(Table 1). This indicates that the interior of the capsule has a much lower $\mathrm{pH}$ than the seawater and the cytoplasm of the surrounding cell. We calculated the internal proton concentration of a cyst on the basis of rather rough estimates of the volume of seawater $(15 \mu \mathrm{l})$, the volume of one cyst $(2.3 \mathrm{pl})$, the number of cysts used in one experiment $(2,500)$, and the buffering capacity of the seawater (to reduce the $\mathrm{pH}$ of $15 \mu \mathrm{l}$ seawater from 7.8 to $6.8,30 \mathrm{nl}$ of $1 \mathrm{M} \mathrm{HCl}$ was necessary). The calculated value was $5 \mathrm{M}$ (estimated error interval from 2.5 to $10 \mathrm{M}$ ). Most protons are contained in protonated carboxyl groups.

When the experiment was performed with acontia isolated about $4 \mathrm{~h}$ before treatment or with acontia isolated from animals fed some hours before treatment, we found a discharge of nematocysts as well, but the $\mathrm{pH}$ only dropped to values just below or still above $\mathrm{pH} 7$. We suggest that only a fraction of the nematocyst population responds very sensitively to external changes such as mechanical handling and contact with prey animals.

We would like to argue against a significant contribution of leakage of protons from lysosomes to the observed decrease of the $\mathrm{pH}$. As shown in Fig. 1b, acontia of Aiptasia consist mainly of nematocysts. Less than half of the acontia consists of other cell components, including cytoplasm, membranes, mitochondria, nuclei, and acidic compartments such as lysosomes. Application of LysoSensor (TM) Blue DND-167 (see later) indicates the almost complete absence of lysosomes from acontia (Fig. 2a, b). The volume of these acidic compartments is much less than one-tenth of the tissue volume of the acontia. If it is assumed that the $\mathrm{pH}$ decrease observed is largely caused by the leakage of protons from these acidic compartments (and not by nematocysts), the proton concentration within these compartments should exceed $20 \mathrm{M}$, an obviously unrealistic assumption.

About 15 young medusae (ephyrae) of the scyphozoon $A$. aurita were placed on the top of a FET. Then the amount of seawater was reduced to $15 \mu \mathrm{l}$, and $40 \mu \mathrm{l}$ of distilled water was added. The $\mathrm{pH}$ dropped to minimally $\mathrm{pH} 6.3$, a value well below that of the cytoplasm (Table 1). We treated in a similar way polyps and medusae of the cubozoan T. cystophora (about 30 polyps and 8 medusae, respectively, per experiment), medusae of C. johnstoni (Hydrozoa) (about 20 animals per
Table 1 Maximal observed decrease of $\mathrm{pH}$ in surrounding water after discharge of nematocysts

\footnotetext{
*Hydra was kept in artificial fresh water. Instead of distilled water, saturated $\mathrm{NaCl}$ solution was applied
}

\begin{tabular}{lll}
\hline Class, species/developmental state or organ & $\begin{array}{l}\mathrm{pH} \text { in seawater } \\
(15 \mu \mathrm{l})\end{array}$ & $\begin{array}{l}\mathrm{pH} \text { after adding } \\
\text { distilled water }(40 \mu \mathrm{l})\end{array}$ \\
\hline Anthozoa, Aiptasia sp./isolated acontia of polyp & 7.7 & 6.4 \\
Scyphozoa, Aurelia aurita/ephyra (young medusa) & 7.8 & 6.3 \\
Cubozoa, Tripedalia cystophora/polyp & 7.9 & 7.0 \\
Cubozoa, Tripedalia cystophora/medusa & 7.9 & 6.8 \\
Hydrozoa, Clytia johnstoni/medusa & 7.8 & 6.7 \\
Hydrozoa, Thecocodium quadratum/medusa & 7.8 & 6.4 \\
Hydrozoa, Cladonema sp./medusa & 7.8 & 6.2 \\
Hydrozoa, Hydra vulgaris/polyp & $7.2^{*}$ & $6.0^{*}$ \\
\hline
\end{tabular}


Fig. 2 Approaches to stain acidic compartments. a, b Living acontia of Aiptasia sp. treated in sea water with LysoSensor (TM) Blue DND167 (1:100 dilution) for $30 \mathrm{~min}$. a Interference contrast, b same preparation, UV-fluorescence. c, d Living ephyrae of Aurelia aurita treated in seawater with LysoSensor (1:100 dilution) for 6 days. c Interference contrast, d same preparation, UVfluorescence. Note that nematocysts generally appear unstained (black) in a fluorescent environment. e, f Crystals of poly-DL-aspartic acid in diluted hydrochloric acid, stained with LysoSensor (1:100 dilution) for $30 \mathrm{~min}$. e Interference contrast, f same preparation, UV-fluorescence. Note that the crystals appear unstained (black) in a

fluorescent environment. $\mathbf{g}$, $\mathbf{h}$ CM-Sephadex equilibrated with $0.5 \mathrm{M}$ sodium phosphate buffer (g) at $\mathrm{pH} 3.0$ and (h) at $\mathrm{pH} 4.0$, treated with LysoSensor
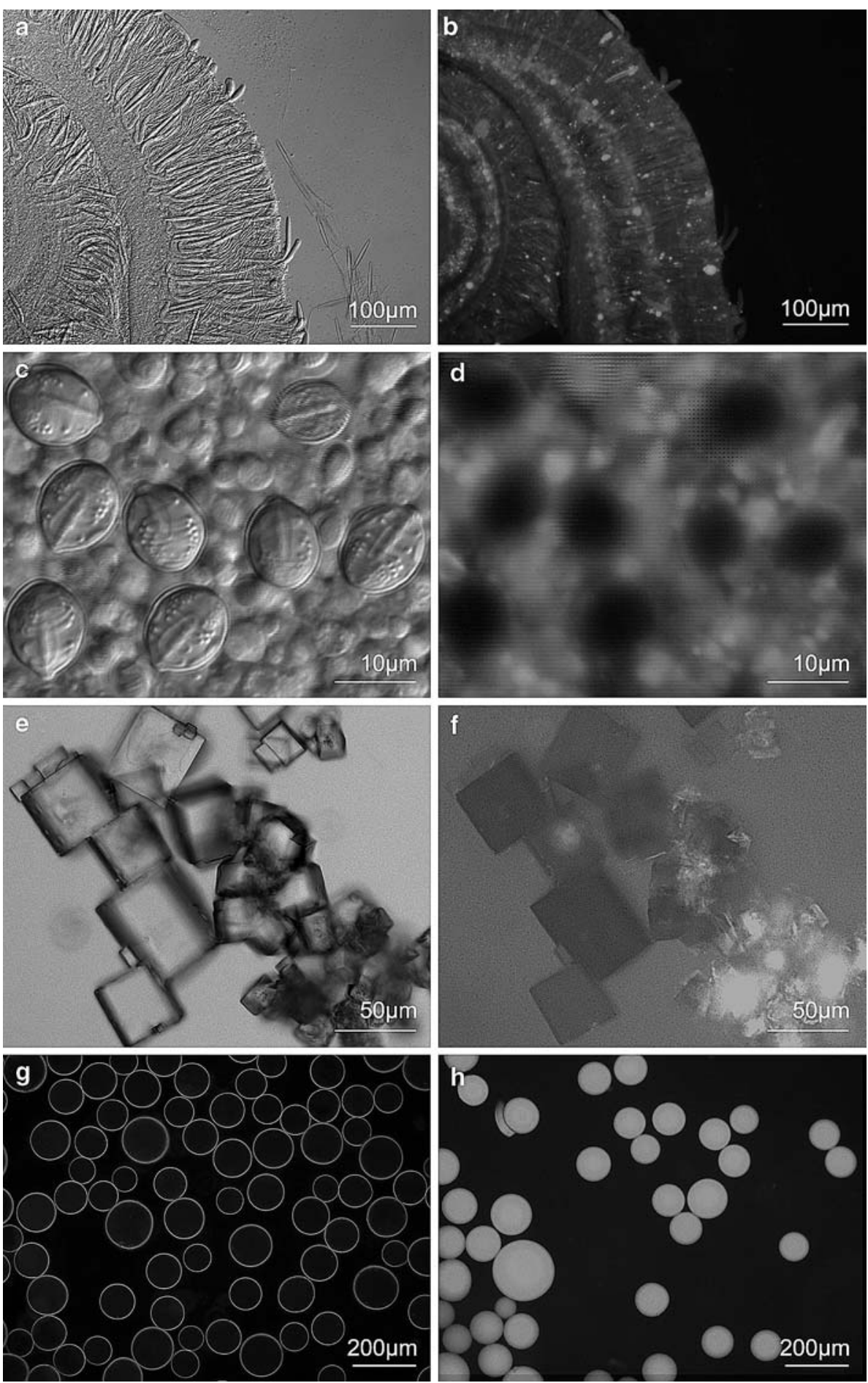

experiment), medusae of $T$. quadratum (Hydrozoa) (about 30 animals per experiment), and medusae of Cladonema sp. (about 12 animals per experiment). In all cases a drop of the $\mathrm{pH}$ was observed (Table 1). Nematocysts of Hydra do not discharge when not treated with distilled water, but when transferred to saturated $\mathrm{NaCl}$ solution. About ten $H$. vulgaris were placed on the top of a FET in about $15 \mu \mathrm{l}$ culture medium. Following the application of 10-20 $\mu$ l saturated $\mathrm{NaCl}$ solution, the pH dropped to minimally 6.0 (Table 1 ).
The minimal $\mathrm{pH}$ reached after discharge was not the same in repetitive experiments with the same species. In the repetitions we used different numbers of animals, and the amount of culture medium per volume of seawater present in the final test volume was certainly not identical. Both seawater and the Hydra culture medium have a buffering capacity. When seawater $(15 \mu \mathrm{l})$ was mixed with distilled water $(40 \mu \mathrm{l})$ and Hydra culture medium $(15 \mu \mathrm{l})$ with saturated $\mathrm{NaCl}$ solution $(40 \mu \mathrm{l})$, we did not observe a fall of the $\mathrm{pH}$. 
In a second set of experiments we used various fluorescent probes to study the matrix of nematocysts. We followed the protocol of Szczepanek et al. (2002) and stained nematocysts of Hydra with Acridin orange (AO) and DAPI (4',6-diamidino-2-phenylindole), respectively. Animals were fixed with formaldehyde in a buffer (pH 7.6) containing EDTA to prevent $\mathrm{Ca}^{2+}$. ions to enter the cyst matrix $\left(\mathrm{Ca}^{2+}\right.$-ions were found to hinder the staining of the cyst matrix). As observed by Szczepanek et al. (2002), the procedure results in a brilliant staining of both the nuclei and the cyst matrix. The color of the nuclei differs from that of the cyst; as in the latter, a metachromic shift in the emission spectrum is caused by binding to poly- $\gamma$-glutamic acid. In another experiment we added the dyes Acridin orange and DAPI, respectively, directly to the culture medium to get a vital staining, and found the nuclei stained immediately but hardly stained the cyst matrix even when the dye had been applied for days. The staining of nuclei indicates that the dyes are able to penetrate unit membranes such as the plasma membrane, whereas the cyst matrix does not appear to be in a condition that allows an enrichment of the dyes. Szczepanek et al. (2002) showed that $\mathrm{Ca}^{2+}$-ions hinder a staining of the cyst matrix, but they also showed that in living animals $\mathrm{Ca}^{2+}$-ions are lacking in the matrix. Thus the results obtained by vital staining call for a different explanation. We suggest that for living animals the $\mathrm{pH}$ of the matrix of nematocysts from the onset of polyacid synthesis onward (see later) is much lower than after fixation at $\mathrm{pH}$ 7.6.

Specimen of Hydra polyps, Aurelia ephyrae, and acontia of Aiptasia were treated with LysoSensor (TM) Blue DND-167. Because of its low $\mathrm{pK}_{a}$ value $\left(\mathrm{pK}_{a}=5.1\right)$ the probe was almost nonfluorescent at neutral $\mathrm{pH}$ and displayed a blue fluorescence in acidic compartments. Our treatment lasted from a few minutes up to 6 days. With increasing time, we found that background staining increased in intensity. A few nematocysts were found to generate a light blue fluorescence (less than $1 \%$ ), whereas the majority appeared black despite having been treated for 6 days; that is, most did not even display the overall background staining (Fig. 2c, d). It appears that the majority of nematocysts do not accumulate the dye. In another experiment we simulated compartments containing polyacids in undissociated form by suspending polyDL-aspartic acid in diluted $\mathrm{HCl}$. After application of the LysoSensor, the solution delivered a strong fluorescence and the precipitated crystals of poly-DLaspartic acid appeared dark (Fig. 2e, f). This shows that undissociated carboxyl groups do not accumulate the dye. We further treated CM-Sephadex beads equilibrated with $0.5 \mathrm{M}$ sodium phosphate buffer at $\mathrm{pH} 3.0$ and $\mathrm{pH} 4.0$, respectively, with the LysoSensor and found that the beads enriched the probe at $\mathrm{pH} 4$ but not at pH 3.0 (Fig. 2g, h). Thus, we propose that in the matrix of nematocysts the carboxyl groups are largely undissociated.

\section{Discussion}

Arguments that nematocysts are acidic inside and display a low osmotic pressure

The current view is that a cyst displays a high internal osmotic pressure and a neutral $\mathrm{pH}$. Nematocysts of various cnidarians contain large amounts of polypeptides rich in glutamic and aspartic acids (Blanquet 1970; Mariscal 1974) including poly- $\gamma$-glutamic acid (pG) (Weber 1990). In stenoteles of Hydra, Weber (1989) found a glutamic acid monomer concentration of about $2 \mathrm{M}$ (chain length of the polymer about 20 units). He argued that the stenoteles are under a constantly high internal osmotic pressure (15.3 MPa) that is dominated by Donnan potential: All small inorganic ions, but not large polyanions, can move unhindered into and out of the capsule. The interior of the capsule was said to display an almost neutral $\mathrm{pH}$, although the $\mathrm{pH}$ value had not been measured. A calculation based on these data and textbooks of physical chemistry leads to an osmotic pressure difference between cyst and cytoplasm in the range of only $0.2-0.5 \mathrm{MPa}$. This calculation is based on a $\mathrm{pG}$ concentration of $0.1 \mathrm{M}(2 \mathrm{M}$ glutamic acid monomer, a mean of 20 units per polymer) $2 \mathrm{M} \mathrm{K}^{+}$(Gerke et al. 1991) and a neutral pH. For reasons of physics the concentration of polymers cannot be much higher. Thus, the contribution of possibly overlooked polymers to the pressure can be ignored. The probability that overlooked low-molecular-weight compounds add to the pressure is low, because they only can exert the pressure to the unit membrane at the outer surface of the capsule but not to the rigid capsule itself. The unit membrane cannot withstand a high pressure. As the cytoplasm contains both small ions and polymers and that $\mathrm{pG}$ is not a strong electrolyte, the osmotic pressure difference at about $\mathrm{pH} 7$ may be closer to 0.2 than to $0.5 \mathrm{MPa}$.

Isolated cysts contain large amounts of $\mathrm{Ca}^{2+}$ (Blanquet 1970, 1988, Weber et al. 1987). Once bound to the matrix, $\mathrm{Ca}^{2+}$ is extremely difficult to remove (Weber et al. 1987). Only in the presence of $\mathrm{Ca}^{2+}$-chelators during fixation it is possible to stain fixed cysts with cationic dyes such as Acridin orange (Szczepanek et al. 2002). It appears that cysts in their natural state do not contain $\mathrm{Ca}^{2+}$. Either the unit membrane prevents the uptake efficiently, or the matrix is in a state unable to enrich $\mathrm{Ca}^{2+}$. We suggest that the latter is more important. Poly- $\gamma$-glutamic acid is argued to be largely protonated and therefore unable to bind $\mathrm{Ca}^{2+}$.

The usual argument for an isolated cyst being still in natural state is its ability to discharge on stimulation. Weber (1989) induced a discharge of isolated cysts that have lost their outer (unit) membrane to discharge by treatment with subtilisin (a protease) or dithioerythritol. This treatment destabilizes the capsule wall. The observed explosion indicates an osmotic pressure difference across the wall and/or mechanical energy stored in the capsule wall and the tubule. However, there is an initial 
volume increase under natural conditions (Holstein and Tardent 1984). This indicates that the discharge is not primarily driven by an existing high internal osmotic pressure or by existing stored mechanical energy, but that on triggering forces are generated that cause an increase in pressure and volume.

What are the arguments for the cyst matrix being acidic? (1) When the discharge is triggered, the surroundings become acidified. For cysts of acontia of Aiptasia, we estimated the internal concentration of protonated carboxyl groups to be about $5 \mathrm{M}$. A contribution of lysosomes to the observed acidification of the surroundings cannot be excluded, but may be ignored because the largest part of the acontia consists of cysts, whereas lysosomes are almost missing. (2) Treatments with AO and DAPI indicate that in the matrix conditions naturally exist that prevent $\mathrm{Ca}^{2+}$-ions to bind to the pG. It seems difficult to find conditions other than a low $\mathrm{pH}$ to explain this finding appropriately. (3) Treatments with AO, DAPI, and LysoSensor did not stain the matrix of cysts in natural state (in vivo). Controls showed that the probes can penetrate membranes and that cysts contain $\mathrm{pG}$, which at neutral condition can accumulate the probes. In this case, too, it appears difficult to find conditions other than a low $\mathrm{pH}$ that could explain these results. We thus propose that cysts contain polyacids that are largely protonated. This fits our findings that CM-Sephadex enriches the LysoSensor at $\mathrm{pH} 4.0$ but not at $\mathrm{pH}$ 3.0. (4) On the basis of a low matrix $\mathrm{pH}$, it is possible to explain that on discharge the volume increases initially. At a low $\mathrm{pH}$ a large number of the carboxyl groups of the polyacids are protonated and therefore uncharged. Two uncharged carboxyl groups of compounds such as $\mathrm{pG}$ can combine each by forming rather stable hydrogen bonds, which causes the formation of aggregates. Apolar interactions between uncharged parts of the molecules further reduce the number of freely diffusible molecules in the cyst, and only such molecules or aggregates contribute to the osmotic pressure. Taking these observations together, we argue that at a low $\mathrm{pH}$ the contribution of the $\mathrm{pG}$ to the matrix osmotic pressure can be ignored. The osmotic pressure in a cyst is almost identical with that of the surrounding cytoplasm.

We speculate that anionic polymers are also present in partly dissociated form in lysosomes and in related vesicles, and serve there as a proton store or buffer. This may explain why a vital staining of cells of various animals with Acridin orange causes lysosomes to appear orange, whereas nuclei appear green (Stockinger 1964). It appears possible that nematocysts have evolved from such vesicles.

\section{Nematocyst formation}

We suggest the following sequence of events (Fig. 3): (1) Both parts of a cyst, tubule and capsule, are Golgi-derived. The tubule forms as an appendix of the capsule.
At the end of tubule formation the interior of both, capsule and tubule, becomes acidified by an import of protons via a proton pump as in other Golgi-derived vesicles such as lysosomes. (2) The acidification causes a protonation of various groups, including carboxyl and amino groups, and of various compounds located in the matrix and at the inner surface of the capsule and the tubule membrane. We suggest that the change of charge of the inner surface affects the conformation of certain membrane components of the tubule in such a way that an invagination is energetically favored. Invagination starts at the distal tip of the tubule. (3) In stenoteles of Hydra, pG synthesis starts when the tubule is taken up (Weber 1990; Szczepanek et al. 2002). Thus pG is not involved in tubule inversion and tubule uptake. The elevated proton concentration causes the protonation of carboxyl groups of the newly synthesized $\mathrm{pG}$ and/or other polyanions and, finally, their dense package and aggregation (Fig. 3). Because of the aggregation of the pG molecules, the pores in the cyst wall can be rather large without causing a loss of $\mathrm{pG}$. The protonation hinders the staining of the matrix with cationic dyes such as Acridin orange (AO), DAPI, and LysoSensor. (4) In general, nematocytes, that is, the cells that house cysts, are produced at sites far away from those where they finally become exposed. In Hydra they are produced in tissue of the gastric region. At the end of differentiation most nematocytes are positioned in the tentacles between and within ectodermal epithelial cells. In most animals, cysts that have not reached their final place do not discharge on weak acidification of the surroundings or on being stimulated by electric currents (Kühn 1916). In some species, during migration the cyst reaches its final size and the wall, its final rigidity. In others, cysts were found to display a soft surface during migration. In particular some of the extremely large cysts in siphonophora produce laces and during migration bend to circumvent obstacles (Will 1910, after Kühn 1916). Obviously the pressure difference across the wall cannot be high, although the synthesis of $\mathrm{pG}$ or related polyacids may have completed. (5) When the final place is reached, cysts that displayed a soft surface up to that time decrease in volume. It may decrease down to half of their previous size (Iwanzoff 1896, Schneider 1900, after Kühn 1916). It appears that in most cases cysts that have not yet reached their final place have not taken their final developmental step. The most important aspect of this step may be a further acidification. This results in a loss of inorganic cations and of water from the cyst and thus in a reduction of the volume.

Most of these findings are difficult to explain on the basis of the current view that the internal pressure exceeds 100 bar. With the first $\mathrm{pG}$ to be synthesized in the matrix, at neutral $\mathrm{pH}$ the pressure exceeds that of the surrounding cytoplasm. This increased pressure should prevent indentations in the wall, a bending during migration and a reduction of volume as a final step of development. Furthermore, one has to explain why an accidental explosion does not occur. In our view 


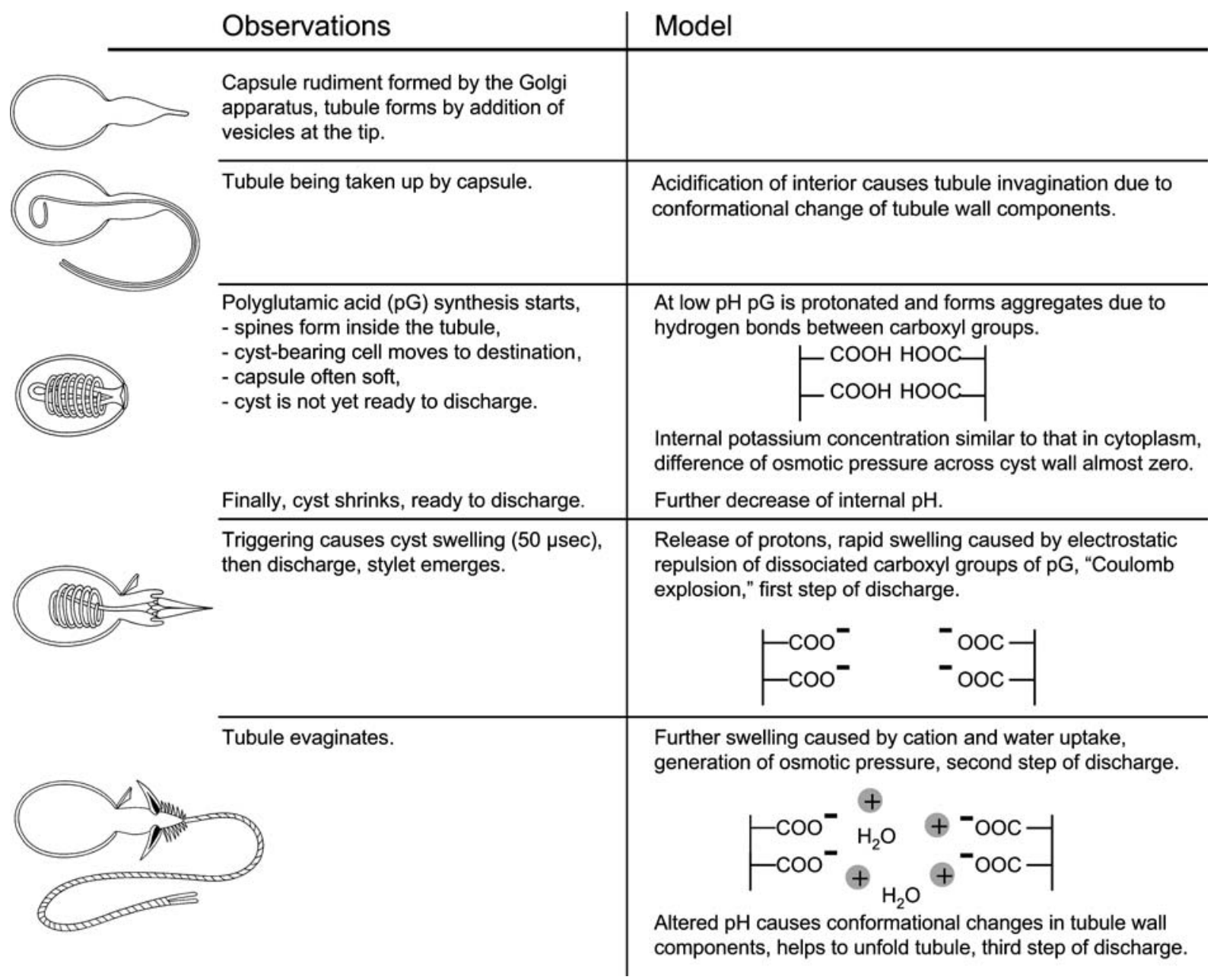

Fig. 3 Formation and discharge of nematocysts is controlled by a proton gradient across the cyst membrane. Figures from Kühn (1916) and Holstein (1981), modified by J. Jacobi. $p G$ polyglutamic acid, a major component of the cyst matrix (Weber 1989, 1990).

cnidarians apply an improved mechanism: the acidification of a subcellular compartment.

\section{Nematocyst discharge}

In a ready-to-discharge cyst the $\mathrm{pH}$ of the cyst matrix is well below the $\mathrm{pH}$ of the cytoplasm of the host cell (Fig. 3). Thus a large number of the carboxyl groups of the polyacids are protonated and therefore uncharged. The osmotic pressure may reach that of the surrounding cytoplasm. Discharge is suggested to start when the membrane can no longer maintain the high internal proton concentration. The leakage of some protons causes (1) an acidification of the surroundings, (2) a change of the membrane potential, and (3) an increased number of dissociated carboxyl groups in the interior of the cyst. Both - an increased proton concentration at the outer surface and an altered membrane potential - may somehow facilitate a further leakage of protons. However, there are several observations that isolated cysts
Indicated onset of pG synthesis after Weber (1990) and Szczepanek et al. (2002). Discharge preceded by cyst swelling according to Holstein and Tardent (1984)

did not discharge when a current was applied or when treated with diluted acids or a concentrated $\mathrm{NaCl}$ solution (Will 1914, after Kühn 1916). It seems these treatments normally act by causing depolarization of the cell membrane, which initiates the loss of protons at the top of the cyst, which in turn results in the opening of the operculum. If the loss starts too slow or at a different site, the operculum may not open properly or even not at all.

At $\mathrm{pH} \mathrm{3,} \mathrm{the} \mathrm{internal} \mathrm{proton} \mathrm{concentration} \mathrm{would} \mathrm{be}$ 10,000 times higher than that of the surroundings. This difference is the driving force of discharge. The movement of protons across the membrane is a rapid process. Protons from the interior of the cyst compensate extremely fast for the loss close to the inner surface of the membrane. In fact, the speed of protons in water is much faster than that of any other cations. This is because in water protons do not simply spread by diffusion but mainly through a shift of charge in chains of water molecules. At acidic conditions polyglutamic acid molecules combine via hydrogen of their protonated car- 
boxyl groups. These groups are therewith close to each other. Because of the loss of protons, the groups dissociate and both aquire a negative charge. This results in an electrostatic repulsion of the $\mathrm{pG}$ molecules, which in turn causes an increase of pressure on the capsular wall and an increase in the capsule volume (Fig. 3). The loss of protons in the cyst's interior and its compensation by dissociation of carboxyl groups is so fast that initially this loss of positive charge is not compensated for by an equivalent uptake of potassium or other cations. We suggest this extremely fast (about $50 \mu \mathrm{sec}$, Holstein and Tardent 1984) volume increase to have features of a socalled Coulomb explosion. We therefore suggest the initial volume increase is not caused by ion and water uptake (this process is much too slow) and thus not by an increased osmotic pressure. In fact, an increased pressure from electrostatic repulsion would cause water to leave the cyst. Thus, in several types of cysts only a sudden pressure increase may be able to cause discharge. If the proton concentration equilibrates only slowly, the loss of water may be quick enough to obviate an increase in cyst volume (see next).

The second step of discharge is caused by the uptake of inorganic cations and water, resulting in an increase of the osmotic pressure and/or cyst volume. The uptake of these two components is much slower than the loss of protons. In cysts that do not discharge very fast, the loss of protons may take too long a time to cause a volume increase. In this case discharge is not caused by electrostatic repulsion but rather by an increasing osmotic pressure. Both the first and the second step together cause a volume increase that is well known to people working with ion exchangers based on carboxyl groups, such as CM-Sephadex C-50: The bead volume almost doubles when the $\mathrm{pH}$ is changed from 3 to 6 at constant ionic strength.

We consider the evagination of the tubule not only to be the result of an increased internal pressure but also to include a conformational change of wall components, driven by the altered $\mathrm{pH}$. Such a change was reported to take place at the outer surface (the former interior) of tubules of discharged cysts: strings in form of spirals ("Quellspiralen") were found to swell on discharge (Will 1914, after Kühn 1916). In addition, several tubules are decorated with rows of barbed spines that are packed tightly before discharge and extend in the surroundings after discharge. Their unfolding may be facilitated by the $\mathrm{pH}$ increase. In Anthozoa some nematocysts were found to have a thin wall and a matrix that could not be stained (Pax 1914). For these nematocysts we hypothesize that the concentration of polyanions is low and that the evagination of the tubule is largely driven by conformational changes of wall components. Thus the speed of discharge should be low. In fact, these nematocysts do not kill a prey but, rather, glue the anthozoan specimen to a substrate.

In summary we suggest that three driving forces are involved in the discharge of nematocysts, all resulting from a loss of protons: electrostatic repulsion, genera- tion of osmotic pressure, and conformational change at the inner tubule's surface. The contribution of these different forces, however, may vary depending on the type of cyst.

Our view of nematocyst formation and discharge may help to explain the occurrence of the so-called kleptocnides, that is, nematocysts produced by cnidarians but exposed closely to the surface of noncnidarians that feed on cnidarians. In certain ciliates, ctenophores, plathelminthes, and mollusks these cysts have been described as functional (for review see Kühn 1916; Bouillon 1993). We suggest that cysts that are fully developed but not yet ready to discharge are incorporated. A supply of ATP may be sufficient to cause the final acidification of the cysts that renders them functional.

Acknowledgments We thank W. Weinhold and G. Firmenich for growing the various species used in the experiments.

\section{References}

Blanquet RS (1970) Ionic effects on discharge of the isolated and in situ nematocysts of the sea anemone Aiptasia pallida: a possible role of calcium. Comp Biochem Physiol 35:451-461

Blanquet RS (1988) The chemistry of cnidae. In: Hessinger DA, Lenhoff HM (eds) The biology of nematocysts. Academic, San Diego, pp 407-425

Bouillon J (1993) Hydropolypes-hydroméduses. In: Grassé P-P, Doumenc D (eds) Traité de Zoologie. Anatomie, Systematique, Biologie Tome iii, Cnidaires Ctenaires Fasciule 2. Masson, Paris, pp 29-416

Gerke I, Zierold K, Weber J, Tardent P (1991) The spatial distribution of cations in nematocytes of Hydra vulgaris. Hydrobiologia 216/217:661-669

Gitter AH, Thurm U (1996) Rapid exocytosis of stenotele nematocysts in Hydra vulgaris. J Comp Physiol A 178:117-124

Golz R (1994) The apical surface of hydrozoan nematocytes: structural adaptions to mechanosensory exocytotic function. $\mathbf{J}$ Morphol 222:49-59

Holstein T (1981) The morphogenesis of nematocysts in Hydra and Forskålia: an ultrastructural study. J Ultrastruct Res 75:276-296

Holstein T, Tardent P (1984) An ultrahigh-speed analysis of exocytosis: nematocyst discharge. Science 223:830-833

Kass-Simon G, Scappaticci AA Jr (2002) The behavioral and developmental physiology of nematocysts. Can J Zool 80:17221794

Kroiher M, Siefker B, Berking S (2000) Induction of segmentation in polyps of Aurelia aurita (Scyphozoa) into medusae and formation of mirror-image medusa anlagen. Int J Dev Biol 44:485-490

Kühn A (1916) Coelenterata (Hohltiere). In: Bronn H G (ed) Dr. H. G. Bronn's Klassen und Ordnungen des Tier-Reichs: wissenschaftlich dargestellt in Wort und Bild. Bd. II: Spongien, Coelenteraten und Echinodermen; Abt. II: Coelenterata (Hohltiere); Abschnitt II: Die Hydromedusen. (Abschnitt 1 and 2 of Bd. II Abt. 2 have continuous pagination). Winter'sche Verlagsbuchhandlung, Leipzig

Mariscal RN (1974) Nematocysts. In: Lenhoff H, Muscatine L (eds) Coelenterate biology: reviews and perspectives. Academic, San Diego, pp 129-178

Pax F (1914) Die Actinien. Erg Fortschr Zool 4:339-642

Stockinger L (1964) Vitalfärbung und Vitalfluorochromierung tierischer Zellen. In: Alfert M, Bauer H, Harding CV (eds) Protoplasmologia. Handbuch der Protoplasmaforschung) Springer, Wien vol 2D1 pp 1-96

Szczepanek S, Cikala M, David CN (2002) Poly- $\gamma$-glutamate synthesis during formation of nematocyst capsules in Hydra. J Cell Sci 115:745-751 
Tardent P, Holstein T (1982) Morphology and morphodynamics of the stenotele nematocyst of Hydra attenuata Pall (Hydrozoa, Cnidaria). Cell Tissue Res 224:269-290

Thomason JC (1991) Cnida discharge and the mechanism of venom delivery in Anemonia viridis (Cnidaria, Actinaria). Hydrobiologia 216/217:649-654

Thurm U, Brinkmann M, Golz R, Holtmann M, Oliver D, Sieger T (2004) Mechanoreception and synaptic transmission of hydrozoa nematocytes. Hydrobiologia 530/531:97-105

Weber J (1989) Nematocysts (stinging capsules of Cnidaria) as Donnan-potential-dominated osmotic systems. Eur J Biochem $184: 465-476$
Weber J (1990) Poly (- $\gamma$-glutamic acid)s are the major constituents of nematocysts in Hydra (Hydrozoa, Cnidaria) $\mathrm{J}$ Biol Chem 265:9664-9669

Weber J, Klug M, Tardent P (1987) Detection of high concentration of $\mathrm{Mg}$ and $\mathrm{Ca}$ in nematocysts of various cnidarians. Experientia 43:1022-1025

Zeretzke S, Pérez F, Velden K, Berking S (2002) $\mathrm{Ca}^{2+}$-ions and pattern control in Hydra. Int $\mathrm{J}$ Dev Biol 46:705-710 